TRANSACTIONS OF THE

AMERICAN MATHEMATICAL SOCIETY

Volume 350, Number 9, September 1998, Pages 3815-3835

S 0002-9947(98)02254-5

\title{
OPERATIONS AND SPECTRAL SEQUENCES. I
}

\author{
JAMES M. TURNER
}

\begin{abstract}
Using methods developed by W. Singer and J. P. May, we describe a systematic approach to showing that many spectral sequences, determined by a filtration on a complex whose homology has an action of operations, possess a compatible action of the same operations. As a consequence, we obtain W. Singer's result for Steenrod operations on Serre spectral sequence and extend A. Bahri's action of Dyer-Lashof operations on the second quadrant Eilenberg-Moore spectral sequence.
\end{abstract}

\section{INTRODUCTION}

Overview. This is intended to be the first in a series of papers to address the question of finding a systematic approach for determining when spectral sequences possess a "good" action of operations, provided it comes from a filtration on a chain complex whose homology has an appropriate action of operations. In this paper, we focus on those filtrations which give us either second quadrant homology spectral sequences or first quadrant cohomology spectral sequences. Since the latter type can be interpreted as a third quadrant homology spectral sequence, we can capture both of these notions under the rubric of left plane spectral sequences.

We accomplish our goal, in this situation, by defining the notion of a Dold algebra. This is essentially a chain complex with product which is "commutative up to homotopy". The definition and subsequent methods rely on the algebraic machinery developed by J. P. May in [8]. From this definition, it is easy to see that the homology of a Dold algebra has well defined action of operations. In order to relate this to a spectral sequence coming from a filtration on a Dold algebra, we define the notion of a $\mu$-filtration. We then show that the resulting spectral sequence has a compatible action of operations, defined in a way that captures the type of action originally constructed by W. Singer in [12] and [14].

With this in hand, we then focus our attention on bicomplexes and the spectral sequences arising from the standard filtration on their total complexes. Again, using the approach developed in [12] and [14], we give conditions on the bicomplex so that this standard filtration is a $\mu$-filtration. We then analyze the $E^{2}$-term.

To demonstrate the usefulness of our work, we recover W. Singer's action of Steenrod operations on the Serre spectral sequence, along with all the other applications he makes in [12] and [14]. We also extend A. Bahri's action of Dyer-Lashof operations on the Eilenberg-Moore spectral sequence associated to the pullback

Received by the editors October 21, 1996.

1991 Mathematics Subject Classification. Primary 18G40, 55S05, 55U15; Secondary 18G30, $55 \mathrm{~S} 10,55 \mathrm{~S} 12,55 \mathrm{~T} 10,55 \mathrm{~T} 20$.

Key words and phrases. Spectral sequences, Dold algebras, Steenrod operations, Dyer-Lashof operations, cosimplicial spaces, infinite loop spaces. 
of infinite loop spaces ([1]), and generalize it to the homology spectral sequence associated to a cosimplicial infinite loop space. For further applications see [15].

In the sequel [16] to this paper, we focus on the notion of truncated Dold algebras which serve to model, for example, the structure associated to the total complex for the bicomplex coming from a cosimplicial iterated loop space. We also examine right plane spectral sequences where we model phenomena such as occurs in [7].

Organization of this paper. In section 2 , we review the needed material about chain complexes, filtrations, and spectral sequences. In section 3 , we stipulate what we mean by an action of operations on homology and how it should behave in the spectral sequence coming from a filtration. We then define Dold algebras and $\mu$ filtrations and then show that operations behave well in the associated spectral sequence. All this is then applied, in section 4, to bicomplexes where we give conditions so that the total complex is a $\mu$-filtered Dold algebra. We close the section by examining the $E^{2}$-term. Finally, in section 5, we give our applications.

\section{ACKNOWLEDGMENTS}

The author would like to thank Haynes Miller for suggesting this project, as well as his guidance and advice during the period at M.I.T. this was being worked on, Jim McClure for his interest in this project and for the proof of 5.11, and Bill Singer for sharing an early draft of [14]. Thanks also to Julie Riddleberger for converting this document to LaTeX.

\section{Preliminaries on Chain Complexes}

For simplicity all modules are over $\mathbb{F}_{2}$. By a chain complex we mean a sequence of $\mathbb{F}_{2}$-modules and maps

$$
\cdots \rightarrow C_{i+1} \stackrel{\partial_{i+1}}{\longrightarrow} C_{i} \stackrel{\partial_{i}}{\longrightarrow} C_{i-1} \rightarrow \cdots
$$

for all integers $i$ such that

$$
\partial_{i} \partial_{i+1}=0, \quad i \in \mathbb{Z}
$$

and write $(C, \partial)$ for shorthand (or just $C$ when the boundary maps $\partial_{i}$ are understood). As usual, the homology of $C$ of degree $i$ is defined as

$$
H_{i}(C)=\frac{\operatorname{ker} \partial_{i}}{\operatorname{im} \partial_{i+1}} .
$$

This definition we use in order to capture the classic notion of homology and cohomology in one (see, e.g., [17]). We denote by $\mathbf{C h}$ the category of chain complexes.

We now recall the definition (see, for example, [8]) of a key chain complex, denoted $W$, and review its properties. Let $\pi$ be the group $\left\{1, \sigma: \sigma^{2}=1\right\}$ and $\Lambda=\mathbb{F}_{2}[\pi]$, the group ring of $\pi$ over $\mathbb{F}_{2}$. We then let

$$
W_{j}= \begin{cases}\Lambda\left\langle e_{j}\right\rangle, & j \geq 0 \\ 0, & j<0,\end{cases}
$$

and define $\partial_{i}$ by setting

$$
\partial_{i} e_{i}=(1+\sigma) e_{i-1}, \quad i>0 .
$$


$W$ is in fact a differentially graded coalgebra, that is, there is a map of chain complexes

$$
\Delta: W \rightarrow W \otimes W
$$

making $W$ a (graded) associative coalgebra. We define (2.5) by setting

$$
\Delta e_{m}=\sum_{i+j=m} e_{i} \otimes \sigma^{i} e_{j}
$$

Note that $\Delta$ is a map of chain complexes over $\Lambda$.

Recall that given chain complexes $C$ and $C^{\prime}$ their tensor product $C \otimes C^{\prime}$ is defined by setting

$$
\left(C \otimes C^{\prime}\right)_{m}=\bigoplus_{i+j=m} C_{i} \otimes C_{j}^{\prime},
$$

whose $m^{t h}$ boundary map is $\sum_{i+j=m}\left(\partial_{i} \otimes 1+1 \otimes \partial_{j}\right)$.

If $C$ and $C^{\prime}$ are furthermore chain complexes over $\Lambda$, we define the $\pi$-product $C \underset{\pi}{\otimes} C^{\prime}$ to be the chain complex defined by the coequalizer

$$
C \otimes C^{\prime} \underset{1 \otimes \sigma}{\stackrel{\sigma \otimes 1}{\rightrightarrows}} C \otimes C^{\prime} \rightarrow C \underset{\pi}{\stackrel{2}{\rightrightarrows}} C^{\prime}
$$

An easy calculation shows that $W$ is an acyclic chain complex and that $H_{0} W \cong$ $\mathbb{F}_{2}$. Let $\epsilon: W \rightarrow \mathbb{F}_{2}$ be the induced augmentation. Then $W$ is a $\Lambda$-free resolution of $\mathbb{F}_{2}$.

When filtering a chain complex $C$, it will be of the form

$$
\cdots \subseteq F^{s} C \subseteq F^{s+1} C \subseteq \cdots \subseteq C,
$$

where $s \in \mathbb{Z}$. Given such a filtration there is an associated spectral sequence $\left\{E^{r}, d^{r}\right\}$, bigraded with

$$
E_{m, t}^{0}=\frac{F^{m}}{F^{m-1}}(C)_{m+t},
$$

with $d^{0}$ induced by $\left.\partial\right|_{F^{m} C}$. This shows that

$$
E_{m, t}^{1}=H_{m+t}\left[\frac{F^{m}}{F^{m-1}}(C)\right] .
$$

In general, we can explicitly determine $E^{r}$ as follows: define the bigraded module $Z^{r}$ by

$$
Z_{m, t}^{r}=\left\{x \in F^{m} C_{m+t}: \partial x \in F^{m-r} C\right\} ;
$$

it is then a standard exercise (see [17]) to show that

$$
E_{m, t}^{r}=\frac{Z_{m, t}^{r}}{\left[\partial Z_{m+r-1, t-r+2}^{r-1}+Z_{m-1, t+1}^{r-1}\right]},
$$

and the differential $\partial$ on $C$ induces

$$
d^{r}: E_{m, t}^{r} \rightarrow E_{m-r, t+r-1}^{r} .
$$

Letting $Z_{m, *}^{r}=\bigoplus_{t} Z_{m, t}^{r}$ then $Z_{m, *}^{r+1} \subseteq Z_{m, *}^{r}$ and $Z_{m+1, *}^{r} \subseteq Z_{m, *}^{r+1}$ for all $r$. Set $Z_{m, *}^{\infty}=\bigcap_{r} Z_{m, *}^{r}$ Letting

$$
B_{m, t}^{r}=\partial Z_{m+r-1, t-r+2}^{r-1}+Z_{m-1, t+1}^{r-1}
$$


and $B_{m, *}^{\infty}=\bigcup_{r} B_{m, *}^{r}$ we have

$$
E_{m, *}^{\infty}=\frac{Z_{m, *}^{\infty}}{B_{m, *}^{\infty}}
$$

In order to compare the spectral sequence to $H_{*} C$ we define a filtration $\left\{F^{s} H_{*} C\right\}$ by

$$
F^{s} H_{*} C=\operatorname{ker}\left(H_{*} C \rightarrow H\left(\frac{C}{F^{s} C}\right)\right) .
$$

There is then a map

$$
\lambda: F^{m} H_{m+t} C \rightarrow E_{m, t}^{\infty},
$$

which induces

$$
\bar{\lambda}: \frac{F^{m}}{F^{m-1}}\left(H_{m+t} C\right) \rightarrow E_{m, t}^{\infty} .
$$

In general, we will say that a spectral sequence $\left(E^{r}, d^{r}\right)$ is abutting to $H_{*} C$ if there is a filtration, $\left\{F^{s} H_{*} C\right\}$, together with a map $\lambda$, inducing $\bar{\lambda}$. We say such a spectral sequence converges when $\bar{\lambda}$ is an isomorphism (see [17]).

We note that if $C$ and $C^{\prime}$ are filtered, then $C \otimes C^{\prime}$ is filtered by setting

$$
F^{m}\left(C \otimes C^{\prime}\right)=\sum_{i+j=m} F^{i} C \otimes F^{j} C^{\prime}
$$

Finally, recall that a bicomplex is a doubly $\mathbb{Z}$-graded module $B$ which, for each $s, B_{s, *}$ and $B_{*, s}$ is a chain complex. We denote by $\partial^{h}$ the horizontal differential for $B$ (i.e., $\partial^{h}: B_{s, t} \rightarrow B_{s-1, t}$ ) and by $\partial^{v}$ the vertical differential for $B$ (i.e., $\left.\partial^{v}: B_{s, t} \rightarrow B_{s, t-1}\right)$. Thus $\partial^{h} \partial^{v}=\partial^{v} \partial^{h}$ and $\partial^{h} \partial^{h}=0=\partial^{v} \partial^{v}$.

Given a bicomplex $B$, recall that its total complex $T(B)$ is defined by setting

$$
T(B)_{m}=\prod_{i \geq 0} B_{-i, m+i}
$$

with total differential defined as the formal sum

$$
\partial^{T}=\partial^{h}+\partial^{v}
$$

We filter $T(B)$ by setting

$$
F^{-s} T(B)_{m}=\prod_{i \geq s} B_{-i, m+i}
$$

which we call the natural filtration of $T(B)$.

\section{Operations, Dold Algebras, and Spectral Sequences}

In this section, we focus on chain complexes whose homology possesses an action of operations. In particular, we give conditions so that a chain complex has this property. This is done through the notion of a Dold algebra, the definition of which, and its relationship to operations, is due originally to A. Dold in [6] and generalized by J. P. May in [8]. Once established, we will be able to give conditions for a filtration on a Dold algebra so that a compatible well-behaved action of operations occur on the spectral sequence. This utilizes an approach pioneered by W. Singer in [12] and [14].

Definition 3.1. A chain complex $C$ possesses an action of operations if 
(1) $H_{*} C$ is a graded commutative algebra (not necessarily with unit);

(2) for all integers $i$ there are homomorphisms

$$
Q^{i}: H_{n} C \rightarrow H_{n+i} C
$$

such that

(a) $Q^{i}=0, \quad i<n$,

(b) $Q^{n} x=x^{2}$.

We further call $C$ unstable if, in addition,

(c) $Q^{i}=0, \quad i>0$,

and define, for $i \geq 0$, the Steenrod operation

$$
S q^{i}: H_{n} C \rightarrow H_{n-i} C
$$

by setting

$$
S q^{i}=Q^{-i}
$$

We next make the

Definition 3.2. A Dold algebra is a chain complex $C$ together with a map of chain complexes

$$
\theta: W \underset{\pi}{\otimes}(C \otimes C) \rightarrow C,
$$

where $C \otimes C$ is a $\Lambda$-module by having $\pi$ act by permutation. We denote a Dold algebra by $(C, \theta)$ (or just $C$ when $\theta$ is understood).

Given a Dold algebra $(C, \theta)$ and $m \geq 0$ define a map of graded modules

$$
\theta_{m}: C \otimes C \rightarrow C
$$

of degree $m$, defined by setting

$$
\theta_{m}(x \otimes y)=\theta\left(e_{m} \underset{\pi}{\otimes}(x \otimes y)\right) .
$$

For $m \geq 0$, define the set map

$$
q^{m}: C \rightarrow C
$$

of degree $m$, by setting, for $x \in C_{k}$,

$$
q^{m}(x)=\theta_{m-k}(x \otimes x)+\theta_{m-k+1}(x \otimes \partial x) .
$$

An easy exercise shows that

$$
\partial q^{m}=q^{m} \partial .
$$

Furthermore, one can check that for each $k$ the induced map

$$
Q^{m}: H_{k} C \rightarrow H_{k+m} C
$$

is a homomorphism. Since we have a natural map

$$
H_{*}(C) \otimes H_{*}(C) \rightarrow H_{*}(C \otimes C),
$$

given by $[u] \otimes[v] \rightarrow[u \otimes v]$, then $\theta_{0}$ induces

$$
\mu: H_{*} C \otimes H_{*} C \rightarrow H_{*} C,
$$

which is a commutative product for $H_{*} C$. It is now easy to check that 
Proposition 3.3. Let $(C, \theta)$ be a Dold algebra. Then, using (3.21) and (3.22), $C$ possesses an action of operations. We call this action the induced action of operations.

We now present a useful context under which Dold algebras arise. Let $\mathcal{E}$ and $\mathcal{F}$ be two categories. Given a functor

$$
F: \mathcal{E} \times \mathcal{E} \rightarrow \mathcal{F}
$$

define its twisting to be the functor

$$
F^{\sigma}: \mathcal{E} \times \mathcal{E} \rightarrow \mathcal{F}
$$

given by $F^{\sigma}(C, D)=F(D, C)$. We then say that the group $\pi$ acts on $F$ if there are natural maps

$$
\sigma_{*}: F \leftrightarrow F^{\sigma}: \sigma^{*},
$$

such that $\sigma^{*} \sigma_{*}=1_{F}$ and $\sigma_{*} \sigma^{*}=1_{F^{\sigma}}$.

Given two functors $F, G: \mathcal{E} \times \mathcal{E} \rightarrow \mathcal{F}$ on which $\pi$ acts, then a natural map $f: F \rightarrow G$ is equivariant if the diagram

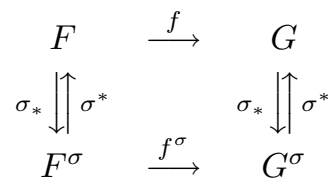

commutes.

Definition 3.4. A triple $(\mathcal{E}, \bar{\otimes}, C)$ is called a complex tensor category if $\mathcal{E}$ is an abelian tensor category (or symmetric monoidal category) with tensor product

$$
\bar{\bigotimes}: \mathcal{E} \times \mathcal{E} \rightarrow \mathcal{E}
$$

and $C: \mathcal{E} \rightarrow \mathbf{C h}$ is a fixed functor.

Example. Let $\mathcal{M}$ be the category of (graded) $\mathbb{F}_{2}$-modules with the usual tensor product $\otimes$. Then the category of simplicial modules $s \mathcal{M}$ becomes a tensor category under the simplicial tensor product $\otimes$ where

$$
(V \bar{\otimes} W)_{s}=V_{s} \otimes W_{s}
$$

The functor $C: s \mathcal{M} \rightarrow \mathbf{C h}$ can be chosen to be the normalization functor, which is an equivalence by the Dold-Kan theorem (see [17]). Unfortunately, $C(V \bar{\otimes} W)$ and $C(V) \otimes C(W)$ are only isomorphic after passing to the homotopy category via the Eilenberg-Zilber theorem. This theorem was extended by Dold in [6] to extract more information from this relationship. We now follow Dold's approach for constructing Dold algebras.

Fix the natural transformation (of graded abelian groups) of degree $q$

$$
\alpha_{q}: \bigotimes \circ(C \times C) \rightarrow W \otimes(\bigotimes \circ(C \times C)),
$$

which is essentially $e_{q} \otimes 1$. Thus $\alpha_{q} \partial=(1 \otimes \partial) \alpha_{q}$.

We now make the 
Definition 3.5. A homotopy deviation, associated to a complex tensor category $(\mathcal{E}, \bar{\otimes}, C)$, is an equivariant chain map

$$
\nabla: W \otimes(\bigotimes \circ(C \times C)) \rightarrow C \circ \bar{\bigotimes}
$$

where $\pi$ acts diagonally on the left, such that:

(1) For each $q$ the natural composite

$$
\begin{gathered}
C(-)_{-q} \otimes C(-)_{-q} \rightarrow(\bigotimes \circ(C \times C))_{-2 q} \stackrel{\alpha_{q}}{\longrightarrow} \\
(W \otimes(\bigotimes \circ(C \times C)))_{-q} \stackrel{\nabla}{\longrightarrow}(C \circ \bar{\bigotimes})_{-q}
\end{gathered}
$$

is a monomorphism.

(2) For any $i, j$ the natural composite

$$
\begin{aligned}
& C(-)_{-i} \otimes C(-)_{-j} \rightarrow(\bigotimes \circ(C \times C))_{-i-j} \stackrel{\alpha_{q}}{\longrightarrow} \\
& (W \otimes(\bigotimes \circ(C \times C)))_{q-i-j} \stackrel{\nabla}{\longrightarrow}(C \circ \bar{\bigotimes})_{q-i-j}
\end{aligned}
$$

is trivial if either $q>i$ or $q>j$.

Let $(E, \mu)$ be a commutative algebroid in a complex tensor category $(\mathcal{E}, \bar{\otimes}, C)$ possessing a homotopy deviation $\nabla$. Then we have a chain map

$$
\theta: W \underset{\pi}{\otimes}(C(E) \otimes C(E)) \rightarrow C(E)
$$

which is induced from the composite

$$
[W \otimes(\bigotimes \circ(C \times C))](E, E) \stackrel{\nabla}{\longrightarrow}(C \circ \bar{\bigotimes})(E, E) \stackrel{C(\mu)}{\longrightarrow} C(E)
$$

since $\nabla$ is equivariant and $\mu$ is commutative. Thus $(C(E), \theta)$ is a Dold algebra and Proposition 3.6. The induced action on $(C(E), \theta)$ gives $C(E)$ an unstable action of operations.

Proof. From above, we just need to check instability, but this follows easily from the definitions and (2) of Definition 3.5.

We now turn to spectral sequences. We will assume, for the remainder of this paper, that a spectral sequence $\left\{E^{r}, d^{r}\right\}$ will be left plane spectral sequence, i.e., $E_{s, *}^{r}=0, s>0$. We thus assume that for any chain complex $C$, a filtration $\left\{F^{s}\right\}$ must be a left filtration, i.e., $F^{s} C=0, s>0$. We now stipulate when operations should behave well with respect to a left plane spectral sequence.

Definition 3.7. Let $C$ be a chain complex possessing an action of operations and $\left\{E^{r}, d^{r}\right\}$ a left plane spectral sequence abutting to $H_{*} C$ with induced filtration $\left\{F^{s} H_{*} C\right\}$. We then call the action of operations well behaved with respect to $\left\{E^{r}, d^{r}\right\}$ if for any $r \geq 2$ there exist homomorphisms

$$
\begin{gathered}
\mu: E_{-m, t}^{r} \otimes E_{-q, u}^{r} \rightarrow E_{-m-q, t+u}^{r} ; \\
Q_{v}^{s}: E_{-m, t}^{r} \rightarrow E_{-m, t+s}^{r},
\end{gathered}
$$

and

$$
Q_{h}^{s}: E_{-m, t}^{r} \rightarrow E_{-m-t+s, 2 t}^{w},
$$

for some $2 r-2 \geq w \geq r$ (see [14]), such that the following hold for a fixed $x \in E_{-m, t}^{r}$. 
(1) $Q_{v}^{s} x=0$, for $s<t, Q_{h}^{s} x=0$, for $s<t-m$ or $s>t$, and $Q_{h}^{t} x=Q_{v}^{t} x$.

(2) Suppose $x$ survives to $[x] \in E^{\ell}, \ell \geq r$. Then $Q_{v}^{s} x$ survives to $E^{\ell}$ and $Q_{v}^{s}[x]=$ $\left[Q_{v}^{s} x\right]$. Further, if $\ell \geq w$, then $Q_{h}^{s} x$ survives to $E^{\ell}$ and $Q_{h}^{s}[x]=\left[Q_{h}^{s} x\right]$. Finally, if $y \in E_{-q, u}^{r}$ survives to $E^{\ell}$, then $\mu(x \otimes y)$ survives to $E^{\ell}$ and $\mu([x] \otimes[y])=$ $[\mu(x \otimes y)]$.

(3) For $d^{r} x \in E_{-m-r, t+r-1}^{r}$ we have that

(a) If $y \in E_{-q, u}^{r}$, then

$$
d^{r} \mu(x \otimes y)=\mu\left(d^{r} x \otimes y\right)+\mu\left(x \otimes d^{r} y\right)+ \begin{cases}Q_{h}^{t-m-1} y, & y=d^{r} x, \\ 0, & \text { otherwise. }\end{cases}
$$

(b) Both $Q_{v}^{s} x$ and $Q_{h}^{s} d^{r} x$ survive to $E^{N}$ and

$$
d^{N} Q_{v}^{s} x=Q_{h}^{s} d^{r} x
$$

where

$$
N= \begin{cases}2 r-1+t-s, & t \leq s \leq t+r-1, \\ 2 r-1, & t-s \leq s \leq t .\end{cases}
$$

(c) If $t+r-1 \leq s$, then $d^{r} Q_{v}^{s} x=Q_{v}^{s} d^{r} x$.

Furthermore, under the map

$$
\lambda: F^{-m} H_{t} C \rightarrow E^{\infty},
$$

we have that for $x \in F^{-m} H_{t} C$ the following holds.

(4) If $y \in F^{-q} H_{u} C$, then $\mu(x \otimes y) \in F^{-m-q} H_{t+u} C$, and $\lambda \mu(x \otimes y)=\mu(\lambda x \otimes \lambda y)$.

(5) For any $s$, if

(a) $t \leq s \leq t+m$, then $Q^{s} x \in F^{-2 m-t+s} H_{t+s} C$ and $\lambda Q^{s} x=Q_{h}^{s} \lambda x$.

(b) If $t+m \leq s$, then $Q^{s} x \in F^{-m} H_{t+s} C$ and $\lambda Q^{s} x=Q_{v}^{s} \lambda x$.

If $\left\{E^{r}, d^{r}\right\}$ is induced by a left filtration $\left\{F^{s} C\right\}$, we call the action of operations well behaved with respect to $\left\{F^{s} C\right\}$ if it is well behaved with respect to $\left\{E^{r}, d^{r}\right\}$.

We now turn to Dold algebras and determine when a filtration is well behaved with respect to the induced action of operations. First, note that if $C$ and $D$ are filtered chain complexes over $\Lambda$, then we filter $C \otimes D$ by letting $F^{m}(C \otimes D)$ be the image of $F^{m}(C \otimes D)$. Next we filter $W$ by setting

$$
\left(F^{j} W\right)_{q}= \begin{cases}W_{q}, & 0 \leq q \leq j ; \\ 0, & \text { otherwise. }\end{cases}
$$

Nonetheless, we use this to define $F^{m}(W \otimes(C \otimes C))$, for any filtered chain complex $C$, as before. We then call a Dold algebra $(C, \theta)$ filtered if there is a filtration $\left\{F^{m} C\right\}$ such that, for each $m, \theta$ induces

$$
\theta: F^{m}(W \underset{\pi}{\otimes}(C \otimes C)) \rightarrow F^{m} C .
$$

Unfortunately, this is insufficient for our needs. To accomplish our goal we define the $\mu$-filtration of $W \underset{\pi}{\otimes}(C \otimes C)$ by setting

$$
\begin{aligned}
F_{\mu}^{m}(W \underset{\pi}{\otimes}(C \otimes C)) \\
\quad=\sum_{i<-m-1} F^{i} W \underset{\pi}{\otimes} F^{m-i}(C \otimes C)+\underset{\pi}{W} F^{2 m+1}(C \otimes C) .
\end{aligned}
$$


We then call a filtered Dold algebra $(C, \theta) \mu$-filtered if for all $m$

$$
\theta\left(F_{\mu}^{m}(W \underset{\pi}{\otimes}(C \otimes C))\right) \subseteq F^{m} C .
$$

We now proceed to prove

Theorem 3.8. Let $(C, \theta)$ be a $\mu$-filtered Dold algebra. Then the induced action of operations on $C$ is well behaved with respect to this filtration.

We first prove

Proposition 3.9. Let $(C, \theta)$ be a $\mu$-filtered Dold algebra. Let $\left\{E^{r}, d^{r}\right\}$ be the associated left plane spectral sequence. Suppose $r \geq 2$ and $x \in Z_{-m, t}^{r}$. Then, if

(1) $t-m \leq s \leq t$, there exists an integer $w$, with $r \leq w \leq 2 r-2$, such that $q^{s} x \in Z_{-m-t+s, 2 t}^{w}$ and the correspondence

$$
x \rightarrow q^{s} x
$$

passes to a homomorphism

$$
Q_{h}^{s}: E_{-m, t}^{r} \rightarrow E_{-m-t+s, 2 t}^{w}
$$

(2) if $t \leq s$, we have $q^{s} x \in Z_{-m, t+s}^{r}$ and the correspondence

$$
x \rightarrow q^{s} x
$$

passes to a homomorphism

$$
Q_{v}^{s}: E_{-m, t}^{r} \rightarrow E_{-m, t+s}^{r}
$$

Proof. (1) Since $x \in Z_{-m, t}^{r}$, then $x \otimes x \in F^{-2 m}(C \otimes C)$. We have $e_{s-t+m} \underset{\pi}{\otimes}(x \otimes x) \in$ $F^{-m-t+s}(W \underset{\pi}{\otimes}(C \otimes C))$; thus $\theta_{s-t+m}(x \otimes x)$ is in $F^{-m-t+s} C$, since $C$ is a filtered Dold algebra. Also, $\partial x \in F^{-m-r} C$, so

$$
\theta_{s-t+m+1}(x \otimes \partial x) \in F^{-m-r-t+s+1} C \subseteq F^{-m+t+s} C, \quad r \geq 1 .
$$

Thus $q^{s} x \in F^{-m-t+s} C$ and by (3.20)

$$
\begin{aligned}
\partial q^{s} x & =\theta_{s+m-t+1}(\partial x \otimes \partial x) \in F^{-m-2 r-t+s+1} C \\
& \subseteq F^{-m-t+s-w} C
\end{aligned}
$$

for any $2 r-2 \geq w \geq r$; hence $q^{s} x \in Z_{-m-t+s, 2 t}^{w}$. By a similar analysis, $w$ can be finessed so that $q^{s} x \in B_{-m-t+s, 2 t}^{w}$. See [14] for further details. 
Next, given $x, y \in Z_{-m, t}^{r}$ we show $q^{s}(x+y)-q^{s} x-q^{s} y$ represents $0 \in E_{-m-t+s, 2 t}^{r}$. Let $k=t-m$,

$$
\begin{aligned}
& q^{s}(x+y)-q^{s} x-q^{s} y=\theta\left(e_{s-k} \otimes_{\pi}(1+\sigma)(x \otimes y)\right)+\theta\left(e_{s-k+1} \otimes_{\pi}(x \otimes \partial y)\right) \\
& +\theta\left(\sigma e_{s-k+1} \underset{\pi}{\otimes}(\partial x \otimes y)\right) \\
& =\theta\left(\partial e_{s-k+1} \underset{\pi}{\otimes}(x \otimes y)\right)+\theta\left(e_{s-k+1} \underset{\pi}{\otimes}(x \otimes \partial y)\right) \\
& +\theta\left(\sigma e_{s-k+1} \underset{\pi}{\otimes}(\partial x \otimes y)\right) \\
& =\partial \theta\left(e_{s-k+1} \underset{\pi}{\otimes}(x \otimes y)\right) \\
& +\theta\left(\partial e_{s-k+1} \otimes_{\pi}(\partial x \otimes y)\right) \\
& =\partial \theta\left(e_{s-k+1} \otimes_{\pi}(x \otimes y)\right) \\
& +\theta\left(e_{s-k+1} \otimes(1+\sigma)(\partial x \otimes y)\right) .
\end{aligned}
$$

One can check, using the fact that $C$ is a $\mu$-filtered Dold algebra, that the last part of the equation is an element of

$$
\partial Z_{-m-t+s+r-1, *}^{r-1}+Z_{-m-t+s-1, *}^{r-1} \quad(r \geq 2)
$$

as required.

(2) This is essentially the same as (1) except that for $s \geq t$ and $x \in Z_{-m, t}^{r}$ we have e.g., $e_{s-t+m} \otimes{ }_{\pi}(x \otimes x) \in F_{\mu}^{-m}(W \underset{\pi}{\otimes}(C \otimes C))$, and so $q^{s} x \in Z_{-m, t+s}^{r}$, as before, and the rest of the proof follows the same path.

Proof of 3.8. One can easily check that since $\theta_{0}: C \otimes C \rightarrow C$ is a map of chain complexes, it induces

$$
\mu: E_{-m, t}^{r} \otimes E_{-q, u}^{r} \rightarrow E_{-m-q, t+u}^{r}
$$

from the definitions. Also the existence of $Q_{h}^{s}$ and $Q_{v}^{s}$ follows immediately from Proposition 3.9. We now confirm the axioms.

(1) This is immediate from Proposition 3.9.

(2) This is also immediate from Proposition 3.9 and the above.

(3)(a) This follows from the definitions and the equation

$$
\partial \theta_{0}(x \otimes y)=\theta_{0}(\partial x \otimes y)+\theta_{0}(x \otimes \partial y) .
$$

(c) Let $u \in E_{-m, t}^{r}$, and $x \in Z_{-m, t}^{r}$ represent $u$. Then if $x \in F^{-m} C$ and $\partial x \in F^{-m-r} C$, since $t \leq s \leq t+r-1$, then $q^{s} \partial x=\theta_{s-t+m+1}(\partial x \otimes \partial x)$ is in $F^{-m-t+s-2 r+1} C=F^{-m-N} C \subseteq F^{-m-r} C$, using the fact that $C$ is a filtered Dold algebra. By (3.20) and the fact that $C$ is a $\mu$-filtered Dold algebra $q^{s} x \in Z_{-m, t+s}^{N}$, and so $Q_{v}^{s} u$ survives to $E^{N}$. Next observe that $Q_{h}^{s} d^{r} u$ is represented by $q^{s} \partial x$ which lies in $Z_{-m-N, *}^{N}$ since $\partial q^{s} \partial x=q^{s} \partial \partial x=0$. Hence $Q_{h}^{s} d^{r} u$ survives to $E^{N}$ and (3.20) tells us that $d^{N} Q_{v}^{s} x=Q_{h}^{s} d^{r} x$. 
Cases (b) and (d) are similar.

(4) and (5). Let $u \in F^{-m} H_{*} C$. Then we can represent $u$ by $x \in F^{-m} C$ such that $\partial x=0$. Thus $q^{s} x=\theta_{s-t}(x \otimes x)$, which is in $F^{-2 m-t+s} C$, for $s \leq t+m$, and in $F^{-m} C$ for $s \geq t+m$, since $C$ is $\mu$-filtered Dold algebra. Thus this result (and the result for $\mu$ ) follows from the definitions.

\section{Bicomplex Tensor Categories}

In this section, we present a context which gives rise to bicomplexes whose total complexes are $\mu$-filtered Dold algebras. We will assume that all our bicomplexes $B$. . are left bicomplexes, that is, $B_{s}$. $=0$ for $s>0$. We denote by $\mathbf{B C h}$ the category of (left) bicomplexes. We also let $\otimes$ and $\bigotimes_{\pi}$ be the obvious generalization to $\mathbf{B C h}$ of tensor product and $\pi$-tensor product that occurs on $\mathbf{C h}$.

Definition 4.1. (1) A (left) bicomplex tensor category is a triple $(\overline{\mathcal{E}}, \hat{\otimes}, B)$ where $(\overline{\mathcal{E}}, \hat{\otimes})$ is a tensor category, with $\overline{\mathcal{E}}$ abelian, and $B: \overline{\mathcal{E}} \rightarrow \mathbf{B C h}$ a fixed functor.

(2) A bicomplex extension of a complex tensor category $(\mathcal{E}, \bar{\otimes}, C)$ is a bicomplex tensor category $(\overline{\mathcal{E}}, \hat{\bigotimes}, B)$ where $\overline{\mathcal{E}}$ is a subcategory of $\operatorname{gr} \mathcal{E}$, the category of graded objects over $\mathcal{E}$, and for $s \in \mathbb{Z}$ we have

a. For $E, E^{\prime} \in \overline{\mathcal{E}}$ then, in $\mathcal{E}$,

$$
\left(E \hat{\otimes} E^{\prime}\right)_{s}=F_{s}\left(E \bar{\otimes} E^{\prime}\right)
$$

where $E \bar{\otimes} E^{\prime}$ is the object of bigr $\mathcal{E}$, the category of bigraded objects over $\mathcal{E}$, such that $\left(E \bar{\otimes} E^{\prime}\right)_{m, t}=E_{m} \bar{\otimes} E_{t}^{\prime}$ and the functor $F_{s}: \operatorname{bigr\mathcal {E}} \rightarrow \mathcal{E}$ is additive exact together with a natural monomorphism $f_{s}: F_{s} \rightarrow \operatorname{diag}_{s}$, where $\operatorname{diag}_{s}:$ bigr $\mathcal{E}_{\rightarrow} \mathcal{E}$ is given by $\operatorname{diag}_{s} E=E_{s, s}$.

b. There is a naturally monic quasi-isomorphism

$$
\varphi_{s}: B \cdot, s \rightarrow C \circ \mathrm{pr}_{s}
$$

where $p r_{s}: g r \mathcal{E} \rightarrow \mathcal{E}$ is given by $\operatorname{pr}_{s}(A)=A_{s}$.

Next we define bicomplexes $W^{h}$ and $W^{v}$ by $W_{*, 0}^{h}=W=W_{0, *}^{v}$ and $W_{*, s}^{h}=0=$ $W_{s, *}^{v}, s \neq 0$, with the differentials induced by the one on $W$. We now make the

Definition 4.2. Let $(\overline{\mathcal{E}}, \hat{\otimes}, B)$ be a bicomplex tensor category.

(1) A Dold complex is a pair $(E, \theta)$ consisting of an object $E \in \overline{\mathcal{E}}$ and a map of bicomplexes

$$
\theta: W_{\pi}^{v} \underset{\pi}{\otimes} B(E \hat{\otimes} E) \rightarrow B(E) .
$$

(2) A complex homotopy deviation is an equivariant map

$$
\gamma: W^{h} \otimes(\bigotimes \circ(B \times B)) \rightarrow B \circ \hat{\bigotimes}
$$

such that, for each $s$, the induced map

$$
W \otimes(\bigotimes \circ(B \times B)) \cdot, s \rightarrow(B \circ \hat{\bigotimes}) \cdot, s
$$

satisfies conditions 1 and 2 of 3.5 
(3) If $\overline{\mathcal{E}}$ is a bicomplex extension of a complex tensor category $(\mathcal{E}, \bar{\otimes}, C)$, with homotopy deviation $\nabla$, then a map of bicomplexes

$$
\gamma: W^{h} \otimes(\bigotimes \circ(B \times B)) \rightarrow B \circ \hat{\bigotimes}
$$

is an extension of $\nabla$ if, for each $s$, the diagram

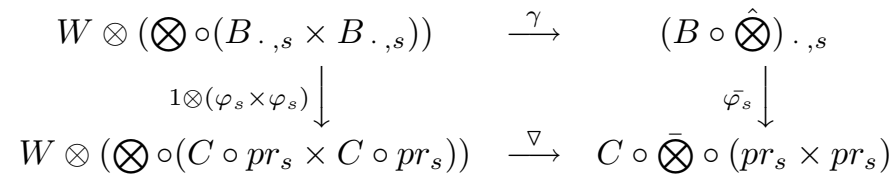

commutes, where $\bar{\varphi}_{s}$ is induced from $\varphi_{s}$ and $f_{s}$, using 2 of 4.1 .

Lemma 4.3. Let $(\overline{\mathcal{E}}, \hat{\otimes}, B)$ be a bicomplex extension of the complex tensor category $(\mathcal{E}, \bar{\otimes}, C)$. Then any extension $\gamma$ of a homotopy deviation $\nabla$ is a complex homotopy deviation.

Proof. This is just a diagram chase using the definitions.

Now, given a functor $B: \mathcal{E} \rightarrow \mathbf{B C h}$ define $T_{B}: \mathcal{E} \rightarrow \mathbf{C h}$ by $T_{B}(E)=T[B(E)]$.

Next, given $A, B$ left bicomplexes there is a natural inclusion

$$
i: T(A) \otimes T(B) \rightarrow T(A \otimes B) .
$$

Indeed, for each $m, n, i$ is given by the composite

$$
\begin{aligned}
\left(\prod_{j} A_{-j, m+j}\right) \otimes\left(\prod_{k} B_{-k, m+k}\right) & \rightarrow \prod_{j, k}\left(A_{-j, m+j} \otimes B_{-k, m+k}\right) \\
& \subseteq \prod_{\ell}(A \otimes B)_{-\ell, m+n+\ell .}
\end{aligned}
$$

Now let $(\overline{\mathcal{E}}, \hat{\otimes}, B)$ be the bicomplex extension of the complex tensor category $(\mathcal{E}, \bar{\otimes}, C)$ and $\gamma$ an extension of a homotopy deviation $\nabla$. Define the twisting of $\gamma$ to be the natural map

$$
\tau(\gamma): W \otimes\left(\bigotimes \circ\left(T_{B} \times T_{B}\right)\right) \rightarrow T \circ\left(W^{v} \otimes(B \circ \bar{\bigotimes})\right)
$$

which for $\left(E, E^{\prime}\right) \in \overline{\mathcal{E}} \times \overline{\mathcal{E}}$ is defined by the composite

$$
\begin{aligned}
& W \otimes T_{B}(E) \otimes T_{B}\left(E^{\prime}\right) \stackrel{\Delta \otimes i}{\longrightarrow} W \otimes W \otimes T\left(B(E) \otimes B\left(E^{\prime}\right)\right) \\
& \stackrel{\phi_{1}}{\longrightarrow} W \otimes T\left(W^{h} \otimes B(E) \otimes B\left(E^{\prime}\right)\right) \stackrel{1 \otimes T(\gamma)}{\longrightarrow} W \otimes T_{B}\left(E \bar{\otimes} E^{\prime}\right) \\
& \stackrel{\phi_{2}}{\longrightarrow} T\left(W^{v} \otimes B\left(E \bar{\otimes} E^{\prime}\right)\right),
\end{aligned}
$$

where $\phi_{1}, \phi_{2}$ are defined in an obvious way.

Lemma 4.4. For $\gamma$ an extension of a homotopy deviation $\nabla$, the twisting $\tau(\gamma)$ is equivariant.

Proof. This is immediate from the definition and properties of the various maps. 
For the rest of this section, we fix a (left) bicomplex extension $(\overline{\mathcal{E}}, \hat{\otimes}, B)$ of the complex tensor category $(\mathcal{E}, \bar{\otimes}, C)$ and $\gamma$ an extension of a homotopy deviation $\nabla$. Let $(E, \bar{\theta})$ be a Dold complex in this bicomplex tensor category. Then define

$$
\theta(\gamma): \underset{\pi}{\otimes}\left(T_{B}(E) \otimes T_{B}(E)\right) \rightarrow T_{B}(E)
$$

as induced by the composite

$$
\begin{array}{r}
\left(W \otimes\left(\bigotimes \circ\left(T_{B} \times T_{B}\right)\right)\right)(E, E) \stackrel{\tau(\gamma)}{\longrightarrow}\left(T \circ\left(W^{v} \otimes(B \circ \hat{\bigotimes})\right)\right)(E, E) \\
\longrightarrow T\left(W^{v} \underset{\pi}{\otimes}(B(E) \bar{\otimes} B(E))\right) \stackrel{T(\bar{\theta})}{\longrightarrow} T_{B}(E),
\end{array}
$$

which is $\pi$-equivariant by Lemma 4.4 .

Theorem 4.5. $\left(T_{B}(E), \theta(\gamma)\right)$ is a $\mu$-filtered Dold algebra with respect to the natural filtration.

Proof. Let $B=B(E)$. Since the filtration is naturally defined, it is sufficient to show that the twisting induces

$$
\tau(\gamma): F_{\mu}^{-m}\left(W \otimes T_{B}(E) \otimes T_{B}(E)\right) \rightarrow F^{-m} T\left(W^{v} \otimes B(E \hat{\otimes} E)\right)
$$

for all $m$. Let $x \otimes y \in F^{-q} T_{B}(E)_{s} \otimes F^{-r} T_{B}(E)_{t}$. Then we may assume $x \in$ $B_{-i, s+i}, i \geq q$ and $y \in B_{-j, t+j}, j \geq r$. Let $e \in W_{p}$, which we may assume is $e_{p}$, by equivariance, for which

$$
\Delta e_{p}=\sum_{a+b=p} e_{a} \otimes \sigma^{a} e_{b}
$$

thus, since $\gamma$ is an extension of $\nabla$, we can write

$$
\tau(\gamma)\left(e_{p} \otimes x \otimes y\right)=\sum_{a+b=p} e_{a} \otimes \nabla\left(\sigma^{a} e_{b} \otimes x \otimes y\right) .
$$

By definition, $\nabla\left(\sigma^{a} e_{b} \otimes x \otimes y\right) \in B(E \bar{\otimes} E)_{-i-j+b, s+t+i+j}$, which is trivial for $b>i$ or $b>j$ by (2) of Definition 3.5, and so is trivial for $q+r+2 b>2 i+2 j$. Thus, since $i+j-b \geq q+r-p$ we have that $\nabla\left(\sigma^{a} e_{b} \otimes x \otimes y\right)$ is in both $F^{-r-q+p} T_{B}(E \bar{\otimes} E)$ and $F^{-\ell} T_{B}(E \hat{\otimes} E)$, where

$$
\ell=\text { least integer greater than } \frac{r+q}{2} .
$$

Now, if $p<m-1$, then $2 p<m+p-1<r+q$ and so $2 r+w q-2 p>r+q$, i.e., $e_{p} \otimes x \otimes y \in F_{\mu}^{-m}\left(W \otimes T_{B}(E) \otimes T_{B}(E)\right)$; otherwise $p \geq m-1$, for which $e_{p} \otimes x \otimes y \in F_{\mu}^{-m}\left(W \otimes T_{B}(E) \otimes T_{B}(E)\right)$ by definition, and by the analysis above we have

$$
\tau(\gamma)\left(e_{p} \otimes x \otimes y\right) \in F^{-m} T\left(W^{v} \otimes B(E \bar{\otimes} E)\right),
$$

since we may assume $q+r=m+p$, and so $q+r \geq 2 m-1$, so that $\ell \geq m$. Conclusion follows.

We now analyze the $E^{2}$-term of the spectral sequence associated to a Dold complex. Recall that for any left bicomplex $B$ the $E^{2}$-term has the form

$$
E_{-m, t}^{2}=H_{-m}^{h} H_{t}^{v}(B)
$$


where $H_{*}^{v}$ denotes homology with respect to $\partial^{h}$. If $E$ is a Dold complex and $B=B(E)$, then for each $m, B_{-m, *}$ is a Dold algebra, and therefore there are chain homomorphisms

$$
\bar{\mu}: H_{q}^{v}(B) \otimes H_{t}^{v}(B) \rightarrow H_{q+t}^{v}(B),
$$

and for each $s$,

$$
\bar{Q}^{s}: H_{m}^{v}(B) \rightarrow H_{m+s}^{v}(B),
$$

which is the induced action of operations on $B_{-m, *}$ for each $m$. Next for each $t, H_{t}(B)$ is an unstable Dold algebra by Proposition 3.6. Thus (4.7) induces

$$
\mu: H_{-m}^{h} H_{t}^{v}(B) \otimes H_{-q}^{h} H_{u}^{v}(B) \rightarrow H_{-m-q}^{h} H_{t+u}^{v}(B),
$$

(4.8) induces

$$
Q^{s}: H_{-m}^{h} H_{t}^{v}(B) \rightarrow H_{-m}^{h} H_{t+s}^{v}(B)
$$

and (4.7) and $h$ induce

$$
S q^{s}: H_{-m}^{h} H_{t}^{v}(B) \rightarrow H_{-m-s}^{h} H_{2 t}^{v}(B) .
$$

Lemma 4.6. For $x \in H_{-m}^{h} H_{t}^{v}(B)$ we have

$$
S q^{s} Q^{\frac{i}{2}} x=Q^{i} S q^{s} x
$$

provided $\bar{Q}_{v}^{k}$ satisfies the Cartan formula on products in $H_{*}^{v}(B)$.

Proof. Since $\bar{Q}^{i}: H_{t}^{v}(B) \rightarrow H_{t+i}^{v}(B)$ is a map of chain complexes, then representing $x$ by $u$ in $H_{t}^{v}(B)$ we have that $S q^{s} x$ is represented by $\bar{\mu} \nabla\left(e_{s-m} \otimes u \otimes u\right)$ in $H_{2 t}^{v}(B)$. By the assumption we compute, using naturality, that

$$
\begin{aligned}
\bar{Q}^{i} \nabla\left(e_{s-m} \otimes u \otimes u\right)= & \sum_{k+\ell=i} \nabla\left(e_{s-m} \otimes \bar{Q}^{k} u \otimes \bar{Q}^{\ell} u\right) \\
= & \nabla\left(e_{s-m} \otimes \bar{Q}^{\frac{i}{2}} u \otimes \bar{Q}^{\frac{i}{2}} u\right) \\
& \quad+\sum_{2 k<i} \nabla\left(e_{s-m} \otimes(1+\sigma)\left(\bar{Q}^{k} u \otimes \bar{Q}^{i-k} u\right)\right) .
\end{aligned}
$$

Therefore, $Q^{i} S q^{s} x$ is represented by

$$
\bar{\mu} \nabla\left(e_{s-m} \otimes \bar{Q}^{\frac{i}{2}} u \otimes \bar{Q}^{\frac{i}{2}} u\right)+\partial \bar{\mu} \nabla\left(e_{s-m+1} \otimes\left(\sum_{k<i} \bar{Q}^{k} u \otimes \bar{Q}^{k} u\right)\right),
$$

which also represents $S q^{s} Q^{\frac{i}{2}} x$.

Theorem 4.7. Let $E$ be a Dold complex. Then in the natural spectral sequence $\left\{E^{r}, d^{r}\right\}$ associated to the Dold algebra $\left(T_{B}(E), \theta(\gamma)\right)$ the induced algebra structure on $E^{2}$ coincides with the one on $H_{*}^{h} H_{*}^{v}(B)$, and for each $x \in E_{-m, t}^{2}$ we have for each $s$,

$$
Q_{h}^{s} x=S q^{t-s} x
$$

and

$$
Q_{v}^{s} x=Q^{s} x
$$


Before we prove this we develop some technical devices. Again we let $B=B(E)$. First define

$$
\mathcal{F}^{j}: B_{-m, t} \otimes B_{-q, u} \rightarrow W \otimes T_{B}(E) \otimes T_{B}(E)
$$

by $\mathcal{F}^{j}(x \otimes y)=e_{j} \otimes x \otimes y$. Then, for any $i+j=p$, let

$$
\tau_{i, j}^{p}: B_{-m, t} \otimes B_{-q, u} \rightarrow\left(W \otimes B(E \hat{\otimes} E)_{-m-q+i, *}\right)_{t+u+j}
$$

be the composite

$$
\begin{aligned}
B_{-m, t} \otimes B_{-q, u} \stackrel{\mathcal{F}^{p}}{\longrightarrow} W & \otimes T_{B}(E) \otimes T_{B}(E) \stackrel{\tau(\gamma)}{\longrightarrow} T\left(W^{v} \otimes B(E \hat{\otimes} E)\right) \\
& \stackrel{\text { projection }}{\longrightarrow}\left(W \otimes B(E \hat{\otimes} E)_{-m-q+i, *}\right)_{t+u+j} .
\end{aligned}
$$

We then define

$$
\theta_{i, j}^{p}: B_{-m, t} \otimes B_{-q, u} \rightarrow B_{-m-q+i, t+u+j}
$$

as $\theta(\gamma) \tau_{i, j}^{p}$. Then $\theta^{p}: T_{B}(E) \otimes T_{B}(E) \rightarrow T_{B}(E)$ satisfies

$$
\theta^{p}=\sum_{i+j=p} \theta_{i, j}^{p} .
$$

Lemma 4.8. Let $u \in E_{-m, t}^{2}$ be represented by $x \in B_{-m, t}$, so that $\partial^{v} x=0$ and $\partial^{h} x=\partial^{v} \omega$, for some $\omega \in B_{-m-1, t+1}$. Then, letting $n=t-m$, if

(1) $t-m \leq s \leq t, Q_{h}^{s} u \in E_{-m-t+s, 2 t}^{2}$ is represented by $\theta_{0, s-n}^{s-n}(x \otimes x)$ in $B_{-m-t+s, 2 t}$.

(2) If $t \leq s, Q_{v}^{s} u \in E_{-m, t+s}^{2}$ is represented by $\theta_{s-n, 0}^{s-n}(x \otimes x)$ in $B_{-m, t+s}$.

Proof. (1) By (2.12) $u=x+y$ where $y \in F^{-m-1} T_{B}(E)$ and $Q_{h}^{s} u$ is represented by $q^{s}(x+y)$, which lies in $\sum_{i \geq m} B_{-i, j}$, which when written out has the form

$$
\begin{aligned}
\theta^{s-n}(x \otimes x) & +\theta^{s-n}((1+\sigma) x \otimes y)+\theta^{s-n+1}(x \otimes \partial y) \\
& +\theta^{s-n+1}(y \otimes \partial x)+\theta^{s-n+1}(x \otimes \partial x)+\theta^{s-n+1}(y \otimes \partial y) \\
& +\theta^{s-n}(y \otimes y) .
\end{aligned}
$$

Now $x \otimes y \in F^{-2 m-1+j} \subseteq F^{-m-t+s-1} T_{B}(E)$, since $j \leq s-n$ and $T_{B}(E)$ is $\mu$-filtered. By a similar argument we have that $\theta_{i, j}^{s-n}(y \otimes y), \theta_{i, j}^{s-n+1}(x \otimes \partial y)$, $\theta_{i, j}^{s-n+1}(y \otimes \partial x), \theta_{i, j}^{s-n+1}(y \otimes \partial y)$, and $\theta_{i, j}^{s-n+1}(x \otimes \partial x)$ all lie in $F^{-m-t+s+1} T_{B}(E)$. Finally, $x \otimes x \in F^{-2 m}\left(T_{B}(E) \otimes T_{B}(E)\right)$ so $\theta_{i, j}^{s-n}(x \otimes x) \in F^{-2 m+j} \subseteq F^{-m-t+s+1} T_{B}(E)$ only for $j<s-n$, and so only $\theta_{0, s-n}^{s-n}(x \otimes x)$ remains to represent $Q_{h}^{s} u$ in $E_{-m-t+s, 2 t}^{2}$.

Case (2) is similar.

Proof of Theorem 4.7. One can easily check that for $u \in E_{-m, t}^{2}=H_{-m}^{h} H_{t}^{v}(B)$ represented by $x \in B_{-m, t}, \partial x=0$, then

$$
\theta_{s-n, 0}^{s-n}(x \otimes x)=\theta^{s-n}(x \otimes x),
$$

where the right-hand side is the Dold algebra structure for $B_{-m, *}$. Letting $[x] \in$ $H_{t}^{v}(B)$ be the associated element, then one can check that 


$$
\theta_{0, s-n}^{s-n}([x] \otimes[x])=\bar{\mu} \nabla\left(e_{s-n} \otimes[x] \otimes[x]\right) .
$$

The result follows from Lemma 4.8 .

\section{Applications}

Recall that a cosimplicial object over a category $\mathcal{E}$ is a sequence of objects $C^{0}, C^{1}, \ldots$, together with maps:

$$
d^{j}: C^{n-1} \rightarrow C^{n}
$$

and

$$
s^{j}: C^{n+1} \rightarrow C^{n},
$$

for $0 \leq j \leq n$ such that

$$
\begin{aligned}
& d^{j} d^{i}=d^{i} d^{j-1}, \quad i<j, \\
& s^{j} d^{i}= \begin{cases}d^{i} s^{j-1}, & i<j, \\
\mathrm{id}, & i=j, j+1, \\
d^{i-1} s^{j}, & i>j+1,\end{cases} \\
& s^{j} s^{j}=s^{i-1} s^{j}, \quad i>j .
\end{aligned}
$$

Suppose $\mathcal{C}=\mathcal{M}$, the category of (possibly graded) $\mathbb{F}_{2}$-modules. Define $\bar{\otimes}$ on $c \mathcal{M}$ by

$$
(M \bar{\otimes} L)^{s}=M^{s} \otimes L^{s}
$$

with diagonal cofaces and codegeneracies. We also define the functor

$$
C: c \mathcal{M} \rightarrow \mathbf{C h}
$$

by setting $C(M)_{n}=M^{-n}$ and $\partial: C(M)_{n+1} \rightarrow C(M)_{n}$ by $\partial=\sum_{j=0}^{n} d^{j}$. We then define the cohomotopy groups of $M$ by

$$
\pi^{n} M=H_{-n} C(M)
$$

for $n \geq 0$.

Definition 5.1. A cosimplicial Eilenberg-Zilber map $\left\{D^{k}\right\}$ is a sequence of natural maps

$$
D^{k}: \bigotimes \circ(C \times C) \rightarrow C \circ \bar{\bigotimes}
$$

each of degree $k$ such that

(1) $D^{k}=0, \quad k<0$;

(2) $D^{0}=$ id $:(\otimes \circ(C \times C))_{0} \rightarrow(C \circ \bar{\otimes})_{0}$;

(3) $k \geq 0, \partial D^{k}+D^{k} \partial=D^{k-1}+\sigma D^{k-1} \sigma$.

In addition, we call $\left\{D^{k}\right\}$ special if for each pair $M, L$ in $c \mathcal{M}$,

(4) $D^{k}: C_{-j}(M) \otimes C_{-i}(L) \rightarrow C_{-j-i+k}(M) \times C_{-j-i+k}(L)$ is trivial if either $k>i$ or $k>j$

(5) $D^{n}: C_{-n}(M) \otimes C_{-n}(L) \rightarrow C_{-n}(M) \times C_{-n}(L)$ is the identity.

Proposition 5.2. There exists a cosimplicial Eilenberg-Zilber map

$\left\{D^{k}\right\}$. In addition, we can choose $\left\{D^{k}\right\}$ to be special. 
Proof. For simplicial vector spaces, Dold, in [6], established the dual result. To obtain the cosimplicial result, the Eilenberg-Mac Lane map exists, by dualizing, for finite-type cosimplicial vector spaces. The general case can be obtained by a colimit argument (see the proof of Lemma 3.2 in [7]) .

We note that for $x \in C_{-s}(M)$ and $y \in C_{-t}(L)$ with $s+t>0$, then

$$
D^{0}(x \otimes y)=d^{s+t} \cdots d^{s+1} x \otimes d^{s-1} \cdots d^{0} y
$$

in $C(M \bar{\otimes} L)_{-s-t}$ (see [3]). Also, by naturality, if we define

$$
N_{-s} M=M^{s} \cap \operatorname{ker} s^{0} \cap \cdots \cap \operatorname{ker} s^{s-1}
$$

as the normalized chain complex, then $\left\{D^{k}\right\}$ defines

$$
D^{k}: N_{-s} M \otimes N_{-t} L \rightarrow N_{-s-t+k}(M \bar{\otimes} L)
$$

for each $k$. We now define a cosimplicial deviation to be a homotopy deviation $\nabla: W \otimes(\bigotimes \circ(C \times C)) \rightarrow C \circ \bar{\otimes}$ for the complex tensor category $(c \mathcal{M}, \bar{\otimes}, C)$. Given a cosimplicial Eilenberg-Zilber map $\left\{D^{k}\right\}$ and $M, N$ in $c \mathcal{M}$ define

$$
\nabla\left(e_{q} \otimes x \otimes y\right)=D^{q}(x \otimes y),
$$

and

$$
\nabla\left(\sigma e_{q} \otimes x \otimes y\right)=\sigma D^{q}(y \otimes x) .
$$

Then one can check from the definitions that

$$
\begin{aligned}
\partial \nabla\left(e_{q} \otimes x \otimes y\right)= & D^{q-1}(x \otimes y)+\sigma D^{q-1}(y \otimes x)+D^{q} \partial(x \otimes y) \\
= & \nabla\left(e_{q-1} \otimes x \otimes y\right)+\nabla\left(\sigma e_{q-1} \otimes x \otimes y\right) \\
& +\nabla\left(e_{q} \otimes \partial(x \otimes y)\right) \\
= & \nabla\left((1+\sigma) e_{q-1} \otimes x \otimes y\right)+\nabla\left(e_{q} \otimes \partial(x \otimes y)\right) \\
= & \nabla\left(\partial e_{q} \otimes x \otimes y\right)+\nabla\left(e_{q} \otimes \partial(x \otimes y)\right) \\
= & \nabla \partial\left(e_{q} \otimes x \otimes y\right) .
\end{aligned}
$$

Thus $\nabla$ is a map of chain complexes and therefore a cosimplicial deviation provided $\left\{D^{k}\right\}$ is special. Conversely, given a cosimplicial deviation $\nabla(5.1)$ and (5.2) defines a special cosimplicial Eilenberg-Zilber map. We sum this up in

Proposition 5.3. (5.1) and (5.2) determines a one-to-one correspondence

$$
\left\{\begin{array}{c}
\text { cosimplicial } \\
\text { deviations }
\end{array}\right\} \longleftrightarrow\left\{\begin{array}{c}
\text { special cosimplicial } \\
\text { Eilenberg-Zilber } \\
\text { maps }
\end{array}\right\} .
$$

Next, let $X$ be a bicosimplicial object over $\mathcal{M}$, i.e., for each $s, X^{\cdot, s}$ and $X^{s, \cdot}$ are cosimplicial objects over $\mathcal{M}$ and the vertical operators commute with the horizontal operators. Let $\left\{D_{h}^{k}\right\}$ be a cosimplicial Eilenberg-Zilber map in the horizontal direction and $\left\{D_{v}^{k}\right\}$ a cosimplicial Eilenberg-Zilber map in the vertical direction. Let bicM be the category of bicosimplicial modules and let $\hat{\otimes}$ and $B$ be the obvious generalizations of $\bar{\otimes}$ and $C$ to this category.

Lemma 5.4. (bicM, $\hat{\otimes}, B)$ is a bicomplex extension of $(c \mathcal{M}, \bar{\otimes}, C)$. 
Proposition 5.5. Suppose $X$ is a bicosimplicial commutative $\mathbb{F}_{2}$-algebra. Then $\left\{D_{v}^{k}\right\}$ makes $X$ a Dold complex in (bic $\left.\mathcal{M}, \hat{\otimes}, B\right)$. Furthermore, the obvious extension of the homotopy deviation associated to $\left\{D_{h}^{k}\right\}$ makes $T_{B}(X)$ a $\mu$-filtered Dold algebra and, at the $E^{2}$-term, the operation

$$
Q^{s}: H_{-m}^{h} H_{t}^{v}(B(X)) \rightarrow H_{-m}^{h} H_{t+s}^{v}(B(X))
$$

is unstable and satisfies the Adem and Cartan relations, as also does

$$
S q^{s}: H_{-m}^{h} H_{t}^{v}(B(X)) \rightarrow H_{-m-s}^{h} H_{2 t}^{v}(B(X)) .
$$

Proof. This follows immediately from Proposition 3.6 and Theorem 4.5, Theorem 4.7 , and the results of [6].

Corollary 5.6 (see [12] and [14]). Suppose $Z$ is a bisimplicial cocommutative $\mathbb{F}_{2}$ coalgebra and $N$ an $\mathbb{F}_{2}$-module. Then the third quadrant spectral sequence $\left\{E^{r}, d^{r}\right\}$ converging to $H^{*}(\operatorname{diag} Z ; N)$ possesses, for each $r$, a differential graded algebra structure and an action of Steenrod operations

$$
S q_{h}^{s}: E_{-m,-t}^{r} \rightarrow E_{-m+t-s,-2 t}^{r},
$$

and

$$
S q_{v}^{s}: E_{-m,-t}^{r} \rightarrow E_{-m,-t-s}^{r}
$$

satisfying all the usual relations and compatible at $E^{\infty}$ with the induced filtration on $H^{*}(\operatorname{diag} Z ; N)$.

Proof. Apply Proposition 5.5 to $\operatorname{Hom}(Z, N)$.

This has various topological applications gotten by starting with a bisimplicial set $Y$ and letting $Z$ be the free bisimplicial module on $Y$. The diagonal $Y \rightarrow Y \times Y$ induces a cocommutative coalgebra structure on $Z$. In this context, Corollary 5.6 applies to the Serre spectral sequence and the bar spectral sequence. For details on this and other applications, see [12] and [14].

Next, let $A$ be a simplicial object over $\mathcal{M}$. Let $N A$ be the Moore complex of $A$, i.e.,

$$
N_{s} A=A_{s} \cap \operatorname{ker} d_{1} \cap \cdots \cap \operatorname{ker} d_{s},
$$

and $\partial=d_{0}$. For example, the chain complex $W$ is a chain homotopy equivalent to $N\left[\mathbb{F}_{2} E \pi\right]$ where $\mathbb{F}_{2} E \pi$ is the free simplicial module on $E \pi$ (see [8]).

Consider now the category $\mathcal{J}$ of infinite loop spaces (where space $=$ simplicial set). By [9], the infinite loop structure on an object $X$ in $\mathcal{J}$ is determined by, among other things, a map $\bar{\theta}: E \pi \underset{\pi}{\times}(X \times X) \rightarrow X$. By the Eilenberg-Zilber theorem (see [17]), there is a natural chain homotopy equivalence

$$
W \otimes N\left(\mathbb{F}_{2} X\right) \otimes N\left(\mathbb{F}_{2} X\right) \rightarrow N\left(\mathbb{F}_{2}(E \pi \times X \times X)\right),
$$

which induces

$$
W \underset{\pi}{\otimes}\left(N\left(\mathbb{F}_{2} X\right) \otimes N\left(\mathbb{F}_{2} X\right)\right) \rightarrow N\left(\mathbb{F}_{2}(E \pi \underset{\pi}{\times}(X \times X))\right) .
$$

Now, define an $E_{\infty}^{2}$-algebra in $s \mathcal{M}$ to be a pair $(M, \theta)$ consisting of a simplicial module $M$ and a map of simplicial modules

$$
\theta: \mathbb{F}_{2}(E \pi) \underset{\pi}{\bar{\otimes}}(M \bar{\otimes} M) \rightarrow M
$$

It is clear that for $X \in \mathcal{J}$ then $\mathbb{F}_{2}[X]$ is an $E_{\infty}^{2}$-algebra. 
Lemma 5.7. For each $M$ an $E_{\infty}^{2}$-algebra, $N(M)$ is a Dold algebra. As a consequence, for $X$ in $\mathcal{J}, N\left(\mathbb{F}_{2} X\right)$ is a Dold algebra.

Now consider $(\operatorname{cs} \mathcal{M}, \hat{\otimes}, B)$, where $\hat{\otimes}$ is the obvious generalization of tensor product from $c \mathcal{M}$ and $s \mathcal{M}$, and

$$
B: c s \mathcal{M} \rightarrow \mathbf{B C h}
$$

is defined by $B(M)=\bar{C}[N(M)]$, where $\bar{C}: c \mathbf{C h} \rightarrow \mathbf{B C h}$ is the prolongation of $C$.

Lemma 5.8. ( $c s \mathcal{M}, \hat{\otimes}, B)$ is a bicomplex extension of $(c \mathcal{M}, \bar{\otimes}, C)$. Furthermore, any cosimplicial deviation induces an extension through the simplicial EilenbergZilber map.

Corollary 5.9. Given a cosimplicial object $Z$ over $\mathcal{J}$ then $\mathbb{F}_{2}(Z)$ is a Dold complex in cs $\mathcal{M}$ and $T_{B}\left(\mathbb{F}_{2} Z\right)$ is a $\mu$-filtered Dold algebra.

Proof. This follows immediately from 5.8 and 4.5.

We now have enough to prove

Theorem 5.10. Let $X$ be a cosimplicial infinite loop space and $\left\{E^{r}, d^{r}\right\}$ the second quadrant spectral sequence associated to the total complex of $B\left(\mathbb{F}_{2} X\right)$. Then $H_{*}\left(X ; \mathbb{F}_{2}\right)$ is a cosimplicial graded commutative $\mathbb{F}_{2}$-algebra with a compatible action of Dyer-Lashof operations (see [5]), and so $\pi^{*} H_{*}\left(X^{*} ; \mathbb{F}_{2}\right)$ is a bigraded commutative algebra possessing an action of the Dyer-Lashof operation

$$
Q^{s}: \pi^{m} H_{t}\left(X^{\prime} ; \mathbb{F}_{2}\right) \rightarrow \pi^{m+s} H_{2 t}\left(X^{\prime} ; \mathbb{F}_{2}\right),
$$

satisfying Adem, Cartan, instability, etc., for all $s$, and the relations of Lemma 4.6. Under the identification $E_{-m, t}^{2}=\pi^{m} H_{t}\left(X^{\prime} ; \mathbb{F}_{2}\right)$, this product and action of operations is well behaved, in the sense of Definition 3.7, with the spectral sequence $\left\{E^{r}, d^{r}\right\}$.

Proof. The action of Dyer-Lashof operations follows from [5] and naturality. By Proposition 3.6 and Proposition 5.3 the action of Steenrod operations occurs and is independent of choice of cosimplicial Eilenberg-Zilber map as shown in [6]. The relationship to the spectral sequence follows from Corollary 5.9, Theorem 4.5, and Theorem 4.7.

Next, recall that given a cosimplicial space $X$ there is an associated total space (see [4]) $\operatorname{Tot}\left(X^{\cdot}\right)$ which for $q \geq 0$,

$$
\operatorname{Tot}\left(X^{\cdot}\right)_{q}=\operatorname{Hom}\left(\Delta^{\cdot} \times \Delta[q], X^{\cdot}\right),
$$

where $\Delta^{\prime}$ is the standard cosimplicial space with $\Delta^{m}=\Delta[m]$. From [2] there is a filtration $\left\{F^{-s} H_{*}\left(\operatorname{Tot}\left(X^{*}\right) ; \mathbb{F}_{2}\right)\right\}$, together with natural maps

$$
\xi: F^{-s} H_{*}\left(\operatorname{Tot}\left(X^{\cdot}\right) ; \mathbb{F}_{2}\right) \rightarrow F^{-s} H_{*} T_{B}\left(\mathbb{F}_{2} X^{\cdot}\right) .
$$

Proposition 5.11. If $X^{\cdot}$ is a cosimplicial infinite loop space, then $\operatorname{Tot}\left(X^{\cdot}\right)$ is an infinite loop space, and for $x \in F^{-m} H_{t}\left(\operatorname{Tot}\left(X^{\cdot}\right) ; \mathbb{F}_{2}\right)$ if

(1) $t \leq s \leq t+m$, then $Q^{s} x \in F^{-2 m-t+s} H_{t+s}\left(\operatorname{Tot}\left(X^{\cdot}\right) ; \mathbb{F}_{2}\right)$ and $\xi\left(Q^{s} x\right)=$ $Q_{h}^{s}(\xi x)$.

(2) $t+m \leq s$, then $Q^{s} x \in F^{-m} H_{t+s}\left(\operatorname{Tot}\left(X^{\cdot}\right) ; \mathbb{F}_{2}\right)$ and $\xi\left(Q^{s} x\right)=Q_{v}^{s}(\xi x)$. 
Proof (sketch). The following proof is due to J. McClure in [10]. Let

$$
D_{2}: s \mathcal{S} \rightarrow s \mathcal{S}
$$

be the functor $D_{2} X=E \pi \times \underset{\pi}{ }(X \times X)$. By [5] it is enough to compute $H_{*}\left(D_{2} \operatorname{Tot}\left(X^{*}\right)\right)$ $=H_{*}\left(\operatorname{Tot}\left(D_{2} X^{*}\right)\right)$ from the spectral sequence. From section 5 of [3] there is a cosimplicial space $\mathbf{A}_{(s, t)}$ for each $t \geq s \geq 0$ which is universal for elements of the $E^{\infty}$-term of the homotopy spectral sequence associated to a cosimplicial space. From their results we have

$$
E_{-l, m}^{2}\left(\mathbf{A}_{(s, t)}\right)= \begin{cases}\mathbb{F}_{2}, & l=s, m=t \\ 0, & \text { otherwise }\end{cases}
$$

and from [2] this spectral sequence converges to show that $\operatorname{Tot}\left(\mathbf{A}_{(s, t)}\right)=S^{t-s}$ up to 2-completion. Thus $H_{n}\left(\operatorname{Tot}\left(D_{2} \mathbf{A}_{s, t}\right)\right)=\mathbb{F}_{2}$ for $n \geq 2(t-s)$ (see [5]). From the universality of $\mathbf{A}_{(s, t)}$ we are reduced to showing that

$$
E_{-l, m}^{2}\left(D_{2} \mathbf{A}_{(s, t)}\right)= \begin{cases}\mathbb{F}_{2}, & 2 s \geq l \geq s, m=2 t, \\ \mathbb{F}_{2}, & l=s, m \geq 2 t \\ 0, & \text { otherwise }\end{cases}
$$

but this is a straightforward computation utilizing the definition of $\mathbf{A}_{(s, t)}$.

As an application, let

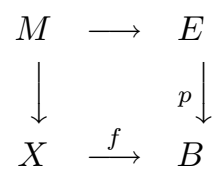

be a fibre square of infinite loop spaces and maps. Then the geometric cobar construction $\mathcal{B}$ is a cosimplicial infinite loop space such that

$$
\mathcal{B}^{s}=X \times B \times \cdots \times B \times E
$$

with $\mathrm{s}$ copies of $\mathrm{B}$ in this product. The resulting homology spectral sequence is the Eilenberg-Moore spectral sequence (see e.g. [2])

$$
E_{-m, t}^{2}=\operatorname{Cotor}_{H_{*} B}^{m}\left(H_{*} X, H_{*} E\right)_{t} \Longrightarrow H_{t-m} M
$$

As a consequence of 5.10 we have operations

$$
Q^{i}: \operatorname{Cotor}_{H_{*} B}^{m}\left(H_{*} X, H_{*} E\right)_{t} \rightarrow \operatorname{Cotor}_{H_{*} B}^{m}\left(H_{*} X, H_{*} E\right)_{t+i}
$$

and

$$
S q^{i}: \operatorname{Cotor}_{H_{*} B}^{m}\left(H_{*} X, H_{*} E\right)_{t} \rightarrow \operatorname{Cotor}_{H_{*} B}^{m+i}\left(H_{*} X, H_{*} E\right)_{2 t}
$$

which is well behaved in the spectral sequence and is compatable with the action of Dyer-Lashof operations on $H_{*} M$, as described by 5.11 .

Note: In [1], the vertical Dyer-Lashof operations were shown to exist in the Eilenberg-Moore spectral sequence for a fibre square of infinite loop spaces. Further, the work of Rector, in [11], and Smith, in [13], shows that the horizontal Steenrod operations exist in the above way. Here we have given them a unified meaning. 


\section{REFERENCES}

[1] A. Bahri, Operations in the second quadrant Eilenberg-Moore spectral sequence J. Pure and Appl. Alg. 27(1983), 207-222 MR 85b:55031

[2] A. K. Bousfield, On the homology spectral sequence of a cosimplicial space, Amer. J. of Math. 109(1987), 361-394. MR 88j:55017

[3] A. K. Bousfield and D. M. Kan, A second quadrant homotopy spectral sequence, Trans. A.M.S. 177(1973), 305-318. MR 51:9063

[4] - Homotopy Limits, Completions, and Localizations, Lecture Notes in Mathematics 304, Springer-Verlag, 1972. MR 51:1825

[5] F. Cohen, T. Lada, and J. May, The Homology of Iterated Loop Spaces, Lecture Notes in Mathematics 533, Springer-Verlag. MR 55:9096

[6] A. Dold, Über die Steenrodschen Kohomologieoperationen, Ann. of Math. 73(1961), 258-294. MR 23:A646

[7] W. Dwyer, Higher divided squares in second quadrant spectral sequences, Trans. A.M.S. 260(1980), 437-447. MR 81f:55022

[8] J. P. May, A general algebraic approach to Steenrod operations, The Steenrod Algebra and its Applications, Lecture Notes in Mathematics 168, Springer-Verlag (1970), 153-231. MR 43:6915

[9] The Geometry of Iterated Loop Spaces, Lecture Notes in Mathematics 271, Springer-Verlag, 1972. MR 54:8623b

[10] J. McClure, Private communication, November 1993.

[11] D. Rector, Steenrod operations in the Eilenberg-Moore spectral sequence, Comment. Math. Helv. 45 (1970), 540-552. MR 43:4040

[12] W. Singer, Steenrod squares in spectral sequences I, II, Trans. A.M.S. 175(1973), 327-336, 337-353. MR 47:7739

[13] L. Smith, On the Kunneth theorem I, Math. Zeit. 116 (1970), 94-140. MR 44:3315

[14] Steenrod squares in spectral sequences: the cohomology of Hopf algebra extensions and of classifying spaces, preprint, Fordham University (1997)

[15] J. Turner, Looping Bousfield-Kan Towers, in preparation

[16] Operations and spectral sequences II, III, in preparation

[17] C. Weibel, An Introduction to Homological Algebra, Cambridge Studies in Advanced Mathematics 38, Cambridge University Press, 1995. MR 95f:18001

Department of Mathematics, College of The Holy Cross, One College Street, Worcester, Massachusetts 01610-2395

Current address: Department of Mathematics, Calvin College, 3201 Burton Street, S.E., Grand Rapids, Michigan 49546-4388

E-mail address: jmt@ziplink.net 